\title{
ON A NONLINEAR BOUNDARY VALUE PROBLEM INVOLVING A SMALL PARAMETER*.
}

\author{
A. ERDÉLYI \\ (received 19 May, 1962)
}

\section{Introduction}

In this paper we shall discuss the boundary value problem consisting of the nonlinear ordinary differential equation of the second order,

$$
\varepsilon y^{\prime \prime}+F\left(t, y, y^{\prime}, \varepsilon\right)=0
$$

$$
0 \leqq t \leqq 1
$$

and the boundary conditions

$$
y(0)=\alpha(\varepsilon), \quad y(1)=\beta(\varepsilon) .
$$

The precise conditions on $F, \alpha, \beta$ are listed in section 4 . Here it will be sufficient to say that $\varepsilon$ is a small positive parameter, $F$ and $\beta$ are continuous functions of $\varepsilon$ at $\varepsilon=0$; we are interested in the behaviour of the solution $y=y(t, \varepsilon)$ as $\varepsilon \rightarrow 0+$; and we envisage circumstances under which $y(t, \varepsilon)$ approaches a limit non-uniformly, the non-uniformity occurring at $t=0$. The boundary value problem consisting of (1.1) and (1.2) will be referred to as the problem $P_{\varepsilon}$.

In some respects $P_{\varepsilon}$ may be regarded as a simple mathematical model of boundary layer phenomena, and accordingly this problem has received some attention. It is not at all easy to formulate conditions under which the existence of a solution can be guaranteed. Coddington and Levinson [1] gave a very simple example of a differential equation, viz.

$$
\varepsilon y^{\prime \prime}+y^{\prime}+y^{\prime 3}=0,
$$

for which the problem $P_{\varepsilon}$ will in general fail to possess a solution for small $\varepsilon$. This failure is generally attributed to the circumstance that $F$ in (1.3) is strongly non-linear in $y^{\prime}$. Accordingly, both Coddington and Levinson [1] and Wasow [2] consider differential equations of the form (1.1) in which $F$ is a linear function of $y^{\prime}$. One of the more interesting results of the discussion to be given below is the replacement of the linearity condition by the condi-

* Most of this research was supported partially by the National Science Foundation under Grant No. NSF G-19914. One phase of the investigations was carried out while the author was the guest of the Summer Research Institute of the Australian Mathematical Society in January 1962; and a report was presented to that Institute. 
tion that $\varepsilon^{-1} \partial^{2} F / \partial y^{\prime 2}$ be bounded. Differential equations (1.1) in which $F$ is a nonlinear function of $y^{\prime}$ were investigated also by Briš: the assumptions. made in section 4 are in some respects more restrictive, and in other respects less restrictive than those made by Briš $[3]$.

If $y(t, \varepsilon) \rightarrow u(t)$ as $\varepsilon \rightarrow 0+, 0<t \leqq 1$, one will expect that $u$ is a solution of the differential equation of the first order

$$
F\left(t, u, u^{\prime}, 0\right)=0 \quad 0 \leqq t \leqq 1 .
$$

Since we envisage circumstances under which the non-uniformity occurs at $t=0$, it will be expected that

$$
u(1)=\beta(0),
$$

but there need be no relation between $\alpha(0)$ and $u(0)$. The boundary value problem consisting of (1.4) and (1.5) will be referred to as the problem $P_{0}$; and it will be one of the basic assumptions that this problem possesses a solution $u$.

Under appropriate conditions it will then be established that there exists a $\mu_{0}>0$, independent of $\epsilon$, so that whenever $|\alpha(\varepsilon)-u(0)|<\mu_{0}$, and $\beta(\varepsilon)=$ $\beta(0)+O(\varepsilon)$, the problem $P_{\varepsilon}$ possesses a unique solution $y=y(t, \varepsilon)$ for all sufficiently small positive $\epsilon$. Furthermore, this solution is of the form

$$
y=u+v+w,
$$

where $v(t)$ and $v^{\prime}(t)$ are $O(\varepsilon)$, and $w(t)$ and $\varepsilon w^{\prime}(t)$ are $O(\exp -\phi(t) / \varepsilon)$, uniformly for $0 \leqq t \leqq 1$, as $\varepsilon \rightarrow 0+$. Here $\phi(t)$ is the non-negative increasing function defined in $(4.1) \equiv$ Clearly, $u+v$ is a uniform approximation to $y$ on $0<\delta \leqq t \leqq 1$ for each positive $\delta$, to $O\left(\varepsilon^{n}\right)$ for any $n$; while $w$ is the boundary layer correction and is negligible except in the immediate neighbourhood of $t=0$.

Our approach is modelled on that of Wasow in that we first construct a solution $y^{*}=u+v$ of (1.1) which satisfies $y^{*}(1)=\beta(\varepsilon)$ and is uniformly close to $u$, and then construct the "boundary layer correction" $w . v$ is constructed by "linearizing" the differential equation (1.1) "around $u$ ", and $w$ is constructed by linearization around $y^{*}$. We differ from Wasow in the technique employed for carrying out these steps. While Wasow employs power series (asymptotic expansions combined with integral equations in the case of $v$, and convergent power series in the case of $w$ ), all our constructions are based directly on integral equation methods. (In the case of $w$ the possibility of such a construction was conjectured, but not used, by Wasow.) As might be expected, a systematic use of integral equation methods leads to a relaxation of differentiability and analyticity conditions. Rather more surprisingly, it also leads to the relaxation of the condition that $F$ be a linear function of $y^{\prime}$. 


\section{The linear equation}

Since (1.1) will be considered as a nonlinear perturbation of a suitable linear approximation, it is desirable to investigate first linear differential equations of the form (1.1). Accordingly, we shall discuss solutions of the differential equation

$$
\varepsilon V^{\prime \prime}+p(t, \varepsilon) V^{\prime}+q(t, \epsilon) V=0 \quad 0 \leqq t \leqq 1 .
$$

Order symbols, such as $O(\varepsilon), o(1)$, refer to $\varepsilon \rightarrow 0+$ and are understood to hold uniformly for $0 \leqq t \leqq 1$.

The discussion of (2.1) will be carried out under the following Assumptions: $p(t, \varepsilon)=\phi^{\prime}(t)+\varepsilon p_{1}(t, \varepsilon), \phi$ is twice continuously differentiable for $0 \leqq t \leqq 1$; $\phi(0)=0, \phi^{\prime}(t)>0 ; p_{1}$ and $q$ are continuous functions of $t$, and are $O(1)$. We also set

$$
p_{2}(t, \varepsilon)=p_{1}(t, \varepsilon)+\frac{\phi^{\prime \prime}(t)}{\phi^{\prime}(t)}=\frac{p(t, \varepsilon)-\phi^{\prime}(t)}{\varepsilon}+\frac{\phi^{\prime \prime}(t)}{\phi^{\prime}(t)} .
$$

The behaviour of the solutions of (2.1) as $\varepsilon \rightarrow 0+$ is essentially known from the asymptotic theory of linear differential equations containing a parameter. Nevertheless, we hall give the derivation of the appropriate results here since a systematic application of integral equation methods enables us to obtain these results under the very slight differentiability assumptions formulated above. For the same reason we shall obtain somewhat more detailed results than are required in the sequel.

Throughout the discussion of the linear equation the following abbreviations will be used:

$$
\begin{aligned}
\psi(t) & =\psi(t, \varepsilon)=\int_{0}^{t} \frac{q(s, \varepsilon)}{\phi^{\prime}(s)} d s, \quad \theta(s, t)=\frac{\phi(s)-\phi(t)}{\varepsilon} \\
\nabla & =\frac{\varepsilon p_{2}}{\phi^{\prime}} V^{\prime}+\psi^{\prime} V .
\end{aligned}
$$

It is easy to verify that any solution of the integro-differential equation

$$
V(t)=1+\int_{0}^{1}\left(1-e^{\theta(\tau, 1)}\right) \vec{V}(\tau) d \tau-\int_{0}^{t}\left(1-e^{\theta(\tau, t)}\right) \vec{V}(\tau) d \tau
$$

satisfies (2.1). Furthermore, it follows from (2.3) and

$$
V^{\prime}(t)=-\frac{\phi^{\prime}(t)}{\varepsilon} \int_{0}^{t} e^{\theta(\tau, t)} \nabla(\tau) d \tau
$$

that $\bar{V}$ satisfies the integral equation 


$$
\begin{aligned}
\vec{V}(t)= & \psi^{\prime}(t)\left[1+\int_{t}^{1} \vec{V}(\tau) d \tau-\int_{0}^{1} e^{\theta(\tau, 1)} \bar{V}(\tau) d \tau\right] \\
& +\left[\psi^{\prime}(t)-p_{2}(t, \varepsilon)\right] \int_{0}^{t} e^{\theta(\tau, t)} \vec{D}(\tau) d \tau .
\end{aligned}
$$

If we denote the right hand side of (2.5) by $T_{1} V$, and regard $T_{1}$ as a transformation of the space of continuous functions of $t, 0 \leqq t \leqq 1$, it turns out that $T_{1}$ is in general (i.e., without further restrictions on $\psi^{\prime}$ ) not a contraction mapping, and the integral equation $\vec{V}=T_{1} \vec{V}$ cannot be solved by successive approximations. We shall decompose $T_{1}$ as $T_{2}+T_{3}$ in such a manner that $1-T_{2}$ possesses an inverse, and $\left(1-T_{2}\right)^{-1} T_{3}=T$ is a contraction mapping. We then re-write $\bar{V}=T_{1} \vec{V}$ in the form $\left(1-T_{2}\right) \vec{V}=T_{3} \vec{V}$ or $\vec{V}=T \vec{V}$, and construct the solution of the latter equation by successive approximations.

We write $(2.5)$ in the form

$$
\begin{aligned}
\nabla(t) & -\psi^{\prime}(t) \int_{t}^{1} \vec{V}(\tau) d \tau=\overline{\bar{V}}(t) \\
& =\psi^{\prime}(t)\left[1-\int_{0}^{1} e^{\theta(\tau, 1)} \bar{\nabla}(\tau) d \tau\right]+\left[\psi^{\prime}(t)-p_{2}(t, \varepsilon)\right] \int_{0}^{t} e^{\theta(\tau, t)} \vec{V}(\tau) d \tau
\end{aligned}
$$

This is equivalent to

$$
-\frac{d}{d t}\left[e^{\psi(t)} \int_{t}^{1} \bar{V}(\tau) d \tau\right]=e^{\psi(t)} \overline{\bar{V}}(t)
$$

and upon integration,

$$
\int_{t}^{1} \bar{V}(\tau) d \tau=\int_{t}^{1} e^{\psi(s)-\psi(t)} \overline{\bar{V}}(s) d s
$$

or

$$
\bar{\nabla}(t)=\overline{\bar{V}}(t)+\psi^{\prime}(t) \int_{t}^{1} e^{\psi(s)-\psi(t)} \overline{\bar{V}}(s) d s
$$

Substituting the definition of $\overline{\bar{V}}$ we finally arrive after some simplification at the integral equation

$$
\vec{\nabla}(t)=T \vec{\nabla}(t),
$$

where $T$ is the functional transformation defined by

$$
\begin{aligned}
T \bar{V}(t)= & \psi^{\prime}(t)\left[1-\int_{0}^{1} e^{\theta(\tau, 1)} \bar{D}(\tau) d \tau\right] e^{\psi(1)-\psi(t)} \\
& +\left[\psi^{\prime}(t)-p_{2}(t, \varepsilon)\right] \int_{0}^{t} e^{\theta(\tau, t)} \bar{D}(\tau) d \tau \\
& +\psi^{\prime}(t) \int_{t}^{1} e^{\psi(s)-\psi(t)}\left[\psi^{\prime}(s)-p_{2}(s, \varepsilon)\right]\left[\int_{0}^{\theta} e^{\theta(\tau, s)} \nabla(\tau) d \tau\right] d s .
\end{aligned}
$$

For a continuous function $f(t)$ we define $\|f\|=\max [|f(t)|: 0 \leqq t \leqq 1]$, and we also choose bounds $A_{i}$ so that for $0 \leqq s, t \leqq 1$ we have 


$$
\begin{aligned}
& \left|\phi^{\prime}(t)\right|^{-1} \leqq A_{1},\left|\psi^{\prime}(t)-p_{2}(t, \varepsilon)\right| \leqq A_{2}, \\
& \left|\psi^{\prime}(t)\right| e^{\psi(3)-\psi(t)} \leqq A_{3}, A_{4}=A_{1}\left(A_{2}+A_{3}+A_{2} A_{3}\right) .
\end{aligned}
$$

Then

$$
\left|\int_{0}^{t} e^{\theta(\tau, t)} f(\tau) d \tau\right| \leqq A_{1}\|f\| \int_{0}^{t} e^{\theta(\tau, t)} \phi^{\prime}(\tau) d \tau<\varepsilon A_{1}\|f\| .
$$

and using this estimate in (2.7), we have for two functions $f_{1}$ and $f_{2}$,

$$
\left\|T f_{1}-T f_{2}\right\| \leqq \varepsilon A_{4}\left\|f_{1}-f_{2}\right\| .
$$

We are now ready to solve the integral equation (2.6) by successive approximations, setting $\nabla_{-1}(t)=0$ and $\vec{V}_{n}=T \nabla_{n-1}$ for $n=0,1,2, \cdots$.

$$
\nabla_{0}(t)=T \nabla_{-1}(t)=\psi^{\prime}(t) e^{\psi(1)-\psi(t)}
$$

from (2.7) so that $\left\|\vec{\nabla}_{0}-\bar{\nabla}_{-1}\right\| \leqq A_{3}$ and from (2.9) we have $\left\|\bar{\nabla}_{n}-\bar{\nabla}_{n-1}\right\| \leqq$ $A_{3}\left(\varepsilon A_{4}\right)^{n}$ by induction. It follows that $\sum_{n=0}^{\infty}\left[\nabla_{n}(t)-\nabla_{n-1}(t)\right]$ converges uniformly provided that $\varepsilon A_{4}<1$. This series defines a function $\nabla(t)$ that satisfies the integral equation (2.6), and hence (2.5), since term by term integration in (2.7) is justified by uniform convergence. Our estimates also show that $\left\|\bar{V}-\bar{\nabla}_{0}\right\| \leqq \varepsilon A_{3} A_{4}\left(1-\varepsilon A_{4}\right)^{-1}$, so that

$$
\nabla(t)=\psi^{\prime}(t) e^{\psi(1)-\psi(t)}+O(\varepsilon)
$$

for the solution of (2.5). Moreover, the solution of (2.6), and hence also the solution of (2.5) and that of the integro-differential equation (2.3), is unique.

3. The results obtained in the preceding section lead directly to some statements on solutions of the differential equation (2.1). To begin with, (2.1) possesses a unique solution $V_{1}$ for which

$$
\begin{aligned}
& V_{1}^{\prime}(0)=0, V_{1}(1)=1, V_{1}(t)=e^{\psi(1)-\psi(t)}+O(\varepsilon), \\
& V_{1}^{\prime}(t)=-\psi^{\prime}(t) e^{\psi(1)-\psi(t)}\left[1-e^{-\theta(t) / \varepsilon}\right]+o(1) .
\end{aligned}
$$

Indeed, $V_{1}$ is the solution of (2.3) and satisfies the boundary conditions given in (3.1). Also,

$$
1+\int_{t}^{1} \nabla(\tau) d \tau=1+\int_{t}^{1} \psi^{\prime}(\tau) e^{\psi(1)-\psi(\tau)} d \tau+O(\varepsilon)
$$

by (2.10), the integrals involving $e^{\theta}$ in (2.3) are $O(\varepsilon)$ by $(2.8)$, and the estimate for $V_{1}$ follows. From (2.4) and (2.10), with (2.8), 


$$
\begin{aligned}
V_{1}^{\prime}(t)= & -\frac{\phi^{\prime}(t)}{\varepsilon} \int_{0}^{t} e^{\theta(\tau, t)} \psi^{\prime}(\tau) e^{\psi(1)-\psi(\tau)} d \tau+O(\varepsilon) \\
= & -\psi^{\prime}(t) e^{\psi(1)-\psi(t)}\left[1-e^{-\phi(t) / \varepsilon}\right]+O(\varepsilon) \\
& +\int_{0}^{t} \frac{\phi^{\prime}(\tau)}{\varepsilon} e^{\theta(\tau, t)}\left[\psi^{\prime}(t) e^{\psi(1)-\psi(t)}-\frac{\phi^{\prime}(t)}{\phi^{\prime}(\tau)} \psi^{\prime}(\tau) e^{\psi(1)-\psi(\tau)}\right] d \tau,
\end{aligned}
$$

and the last integral is $o(1)$ by a well-known result on Laplace integrals since the function in the square brackets approaches zero, uniformly for $0 \leqq t \leqq 1$, as $\tau \rightarrow t$.

Next, we set $V(t)=e^{-\phi(t) / \varepsilon} W(1-t)$ in $(2.1)$ and find that $W$ satisfies the differential equation

$$
\varepsilon W^{\prime \prime}+\left(2 \phi^{\prime}-p\right) W^{\prime}+\left(q-\phi^{\prime} p_{2}\right) W=0
$$

which has the same form as (2.1). Thus we obtain a solution, $W_{1}$, for which statements analogous to (3.1) hold, and correspondingly, there exists a unique solution $V_{2}$ of $(2.1)$ for which

$$
\begin{aligned}
& V_{2}(0)=1, \\
& V_{2}(t)=e^{x(t)-(\phi(t) / \varepsilon)}+O\left(\varepsilon e^{-(\phi(t) / \varepsilon)}\right) \\
& V_{2}^{\prime}(t)=-\frac{\phi^{\prime}(t)}{\varepsilon} e^{x(t)-(\phi(t) / \varepsilon)}+O\left(e^{-(\phi(t) / \varepsilon)}\right),
\end{aligned}
$$

where

$$
\chi(t)=\psi(t)-\int_{0}^{t} p_{2}(\tau, \varepsilon) d \tau .
$$

$V_{1}$ and $V_{2}$ are clearly linearly independent and may be used to construct other solutions. Thus

$$
V_{3}(t)=\frac{V_{1}(1) V_{2}(t)-V_{1}(t) V_{2}(1)}{V_{1}(1) V_{2}(0)-V_{1}(0) V_{2}(1)}
$$

is that (unique) solution of (2.1) satisfying

$$
\begin{aligned}
& V_{3}(0)=1, \quad V_{3}(1)=0 \\
& V_{3}(t)=e^{x(t)-(\phi(t) / \varepsilon)}-e^{\psi(1)-\psi(t)+x(1)-(\phi(1) / \varepsilon)}+O\left(\varepsilon e^{-(\phi(t) / \varepsilon)}\right) \\
& V_{3}^{\prime}(t)=-\frac{\phi^{\prime}(t)}{\varepsilon} e^{x(t)-(\phi(t) / \varepsilon)}+O\left(e^{-(\phi(t) / \varepsilon)}\right) .
\end{aligned}
$$

Furthermore,

$$
K(t, s)=\frac{1}{\varepsilon} \frac{V_{1}(s) V_{2}(t)-V_{1}(t) V_{2}(s)}{V_{1}(s) V_{2}^{\prime}(s)-V_{1}^{\prime}(s) V_{2}(s)}
$$

satisfies 


$$
K(s, s)=0, \quad \varepsilon \frac{\partial K}{\partial t}(s, s)=1
$$

$$
\begin{aligned}
& \phi^{\prime}(s) K(t, s)=-e^{\chi(t)-\chi(s)+\theta(s, t)}[1+O(\varepsilon)]+e^{\psi(s)-\psi(t)}+O(\varepsilon) \\
& \phi^{\prime}(s) \frac{\partial K}{\partial t}(t, s)=\frac{\phi^{\prime}(t)}{\varepsilon} e^{x(t)-\chi(s)-\theta(s, t)}[1+O(\varepsilon)]-\psi^{\prime}(t) e^{\psi(s)-\psi(t)}+o(1) .
\end{aligned}
$$

Let $T_{1}$ and $T_{3}$ be the functional transformations of $C[0,1]$ defined by

$$
\begin{aligned}
& T_{1} f(t)=\left[\lambda-\int_{0}^{1} K(1, s) f(s) d s\right] V_{1}(t)+\int_{0}^{t} K(t, s) f(s) d s \\
& T_{3} f(t)=\left[\mu+\int_{0}^{1} K(0, s) f(s) d s\right] V_{3}(t)-\int_{t}^{1} K(t, s) f(s) d s .
\end{aligned}
$$

$T_{1} f$ and $T_{3} f$ are those solutions of the inhomogeneous differential equation

$$
\varepsilon v^{\prime \prime}+p v^{\prime}+q v=f
$$

satisfying the boundary conditions $\left(T_{1} f\right)^{\prime}(0)=0, T_{1} f(1)=\lambda_{;} T_{3} f(0)=\mu$, $T_{3} f(1)=0$. It can be deduced from (3.1), (3.4) and (3.6) that there exist constants $A_{5}$ and $A_{6}$ independent of $\varepsilon$ and such that for sufficiently small $\varepsilon$,

$$
\begin{gathered}
\left\|T_{1} f_{1}-T_{1} f_{2}\right\|, \quad\left\|\left(T_{1} f_{1}-T_{1} f_{2}\right)^{\prime}\right\| \leqq A_{5}\left\|f_{1}-f_{2}\right\| \\
\left\|e^{\phi / \varepsilon} T_{3} f_{1}-T_{3} f_{2}\right\|, \quad\left\|\varepsilon e^{\phi / \varepsilon}\left(T_{3} f_{1}-T_{3} f_{2}\right)^{\prime}\right\| \leqq A_{6}\left\|e^{\phi / \varepsilon}\left(f_{1}-f_{2}\right)\right\| .
\end{gathered}
$$

It will be sufficient to indicate the proof of (3.10).

$$
\begin{aligned}
& \left|e^{\phi(t) / \varepsilon} \int_{t}^{1} K(t, s) f(s) d s\right| \\
& \quad \leqq\left\|f(t) e^{\phi(t) / \varepsilon}\right\| \int_{t}^{1}|K(t, s)| e^{\theta(t, s)} d s
\end{aligned}
$$

and the last integral is $O(1)$ by (3.6). Similarly,

$$
e^{\phi(t) / \varepsilon} V_{3}(t) \int_{0}^{1} K(0, s) f(s) d s=\left\|f(t) e^{\phi(t) / \varepsilon}\right\| O(1)
$$

by (3.4) and (3.6), and hence

$$
\left[T_{3} f_{1}(t)-T_{3} f_{2}(t)\right] e^{\phi(t) / \varepsilon}=\left\|\left[f_{1}(t)-f_{2}(t)\right] e^{\phi(t) / \varepsilon}\right\| O(1) .
$$

The result for the derivative follows similarly from

$$
\left(T_{3} f\right)^{\prime}(t)=\left[\mu+\int_{0}^{1} K(0, s) f(s) d s\right] V_{3}^{\prime}(t)-\int_{t}^{1} \frac{\partial K}{\partial t}(t, s) f(s) d s
$$

\section{The results}

Let us now return to the non-linear differential equation (1.1) and formulate our assumptions. Partial derivatives will be indicated by subscripts, thus $F_{y}=\partial F / \partial y$; and order symbols like $O(\varepsilon)$ or $O(1)$ will be understood to 
hold as $\varepsilon \rightarrow 0+$, uniformly in all other variables.

The solution of $P_{0}$ will be denoted by $u$, and for this solution we define $\phi$ by

$$
\phi(t)=\int_{0}^{t} F_{\nu^{\prime}}\left(\tau, u(\tau), u^{\prime}(\tau), 0\right) d \tau .
$$

For $\delta>0$ we also introduce $D_{\delta}$, that set of quadruplets $\left(t, y, y^{\prime}, \varepsilon\right)$ for which

$$
0 \leqq t \leqq 1,|y-u(t)|<\delta,\left|y^{\prime}-u^{\prime}(t)\right|<\delta\left(1+\frac{1}{\varepsilon} e^{-(\phi(t) / \varepsilon)}\right), 0<\varepsilon<\varepsilon_{0} .
$$

\section{Assumptions.}

(A) The problem $P_{0}$ consisting of (1.4) and (1.5) possesses a solution $u$ which is twice continuously differentiable for $0 \leqq t \leqq 1$.

(B) For some $\delta>0, F$ is defined in $D_{\delta}$ and possesses there partial derivatives of the first and second orders with respect to $y$ and $y^{\prime}$. Also, $F$ and these partial derivatives are continuous functions of $t, y, y^{\prime}$ (for fixed $\varepsilon$ ).

(C) $F\left(t, u(t), u^{\prime}(t), \varepsilon\right)=O(\varepsilon) ; \quad q(t, \varepsilon)=F_{v}\left(t, u(t), u^{\prime}(t), \varepsilon\right)=O(1) ; \quad F_{v^{\prime}}$ $\left(t, u(t), u^{\prime}(t), 0\right)$ is positive and continuously differentiable for $0 \leqq t \leqq 1$, $F_{y^{\prime}}\left(t, u(t), u^{\prime}(t), \varepsilon\right)=p(t, \varepsilon)=\phi^{\prime}(t)+\varepsilon p_{1}(t, \varepsilon)$, where $\phi$ is defined in (4.1), $p_{1}$ is a continuous function of $t$, and $p_{1}(t, \varepsilon)=O(1) ; F_{y y}\left(t, y, y^{\prime}, \varepsilon\right)=O(1)$, $F_{y y^{\prime}}\left(t, y, y^{\prime}, \varepsilon\right)=O(1), F_{y^{\prime} y^{\prime}}\left(t, y, y^{\prime}, \varepsilon\right)=O(\varepsilon)$.

(D) $\beta(\varepsilon)-\beta(0)=O(\varepsilon)$.

(E) $F_{y}\left(t, y, y^{\prime}, \varepsilon\right)=O(1), F_{y^{\prime}}\left(t, y, y^{\prime}, \varepsilon\right) \geqq B>0$ in $D_{\delta}$.

It follows from these assumptions that $\phi, p, q$ as defined in (C) satisfy the conditions imposed on these functions in section 2 .

THEOREM. Under assumptions (A) to (D) there exists a positive $\mu_{0}$ independent of $\varepsilon$ so that whenever $\varepsilon$ is sufficiently small and $|\alpha(\varepsilon)-u(0)|<\mu_{0}$, the problem $P_{\varepsilon}$ possesses a solution $y$ in $D_{\delta}$. This solution is of the form $y=u+v+w$, where $u$ is the solution of $P_{0}, v(t)$ and $v^{\prime}(t)$ are $O(\varepsilon)$, and $w(t)$ and $\varepsilon w^{\prime}(t)$ are $O(\exp -\phi(t) / \varepsilon)$. Under the additional assumption $(\mathrm{E})$, $y$ is the only solution of $P_{\varepsilon}$ in $D_{\delta}$.

\section{The functions $G$ and $G^{*}$}

Let

$$
\begin{aligned}
G\left(t, z, z^{\prime}, \varepsilon\right)= & -F\left(t, u(t)+z, u^{\prime}(t)+z^{\prime}, \varepsilon\right)-\varepsilon u^{\prime \prime} \\
& +F_{y}\left(t, u(t), u^{\prime}(t), \varepsilon\right) z+F_{v^{\prime}}\left(t, u(t), u^{\prime}(t), \varepsilon\right) z^{\prime},
\end{aligned}
$$

where 


$$
|z|<\delta,\left|z^{\prime}\right|<\delta\left(1+\frac{1}{\varepsilon} e^{-(\phi(t) / \varepsilon)}\right) .
$$

Clearly,

$$
G(t, 0,0, \varepsilon)=O(\varepsilon)
$$

by assumptions (A) and (C). We wish to show that

$$
\begin{aligned}
\mid G\left(t, z_{1}, z_{1}^{\prime}, \varepsilon\right) & -G\left(t, z_{2}, z_{2}^{\prime}, \varepsilon\right) \mid \\
& \leqq k \sigma\left(z_{1}, z_{2}, z_{1}^{\prime}, z_{2}^{\prime}\right),
\end{aligned}
$$

where $k$ is a constant, independent of $t, \varepsilon$ and the $z_{i}, z_{i}^{\prime}$, while $\sigma\left(z_{1}, z_{2}, z_{1}^{\prime}, z_{2}^{\prime}\right)$ is the largest of the two quantities

and

$$
\left|z_{1}-z_{2}\right| \max \left(\left|z_{1}\right|,\left|z_{2}\right|,\left|z_{1}^{\prime}\right|,\left|z_{2}^{\prime}\right|\right)
$$

$$
\left|z_{1}^{\prime}-z_{2}^{\prime}\right| \max \left(\left|z_{1}\right|,\left|z_{2}\right|, \varepsilon\left|z_{1}^{\prime}\right|, \varepsilon\left|z_{2}^{\prime}\right|\right) \text {. }
$$

By the mean value theorem,

$$
\begin{aligned}
G\left(t, z_{1}, z_{1}^{\prime}, \varepsilon\right) & -G\left(t, z_{2}, z_{2}^{\prime}, \varepsilon\right) \\
& =F\left(t, u(t)+z_{2}, u^{\prime}(t)+z_{2}^{\prime}, \varepsilon\right)-F\left(t, u(t)+z_{1}, u^{\prime}(t)+z_{1}^{\prime}, \varepsilon\right) \\
& +F_{y}\left(t, u(t), u^{\prime}(t), \varepsilon\right)\left(z_{1}-z_{2}\right)+F_{v^{\prime}}\left(t, u(t), u^{\prime}(t), \varepsilon\right)\left(z_{1}^{\prime}-z_{2}^{\prime}\right) \\
& =\left[F_{y}\left(t, u(t)+\tilde{z}, u^{\prime}(t)+\tilde{z}^{\prime}, \varepsilon\right)-F_{v}\left(t, u(t), u^{\prime}(t), \varepsilon\right)\right]\left(z_{2}-z_{1}\right) \\
& +\left[F_{v^{\prime}}\left(t, u(t)+\tilde{z}, u^{\prime}(t)+\tilde{z}^{\prime}, \varepsilon\right)-F_{v^{\prime}}\left(t, u(t), u^{\prime}(t), \varepsilon\right)\right]\left(z_{2}^{\prime}-z_{1}^{\prime}\right),
\end{aligned}
$$

where $\tilde{z}=\lambda z_{1}+(1-\lambda) z_{2}, \tilde{z}^{\prime}=\lambda z_{1}^{\prime}+(1-\lambda) z_{2}^{\prime}$ for some $\lambda, 0 \leqq \lambda \leqq 1$. We apply the mean value theorem once more to each of the square brackets and obtain

$$
\begin{aligned}
G\left(t, z_{1}, z_{1}^{\prime}, \varepsilon\right) & -G\left(t, z_{2}, z_{2}^{\prime}, \varepsilon\right) \\
& =F_{v y} \tilde{z}\left(z_{2}-z_{1}\right)+F_{y_{y}}, \tilde{z}^{\prime}\left(z_{2}-z_{1}\right) \\
& +F_{y^{\prime}} \tilde{z}\left(z_{2}^{\prime}-z_{1}^{\prime}\right)+F_{y^{\prime} y^{\prime}} \tilde{z}^{\prime}\left(z_{2}^{\prime}-z_{1}^{\prime}\right) .
\end{aligned}
$$

Here $F_{v v}, \cdots, F_{y^{\prime} y^{\prime}}$ are evaluated at certain intermediate points (different in the first two terms from those in the last two). Since these intermediate points are all in $D_{\delta}$, assumption (C) shows that there is a constant $k$ so that $4\left|F_{y y}\right| \leqq k, 4\left|F_{y y^{\prime}}\right| \leqq k, 4\left|F_{y^{\prime} y^{\prime}}\right| \leqq k \varepsilon$. Since also $|\tilde{z}| \leqq \max \left(\left|z_{1}\right|,\left|z_{2}\right|\right)$ and $\left|\tilde{z}^{\prime}\right| \leqq \max \left(\left|z_{\mathbf{1}}^{\prime}\right|,\left|z_{2}^{\prime}\right|\right)$, this proves (5.4).

Next, let $y^{*}=y^{*}(t)=y^{*}(t, \varepsilon)$ be a continuously differentiable function of $t$ for $0 \leqq t \leqq 1$ and such that $\left(t, y^{*}(t), y^{*}(t), \varepsilon\right)$ is in $G_{\delta / 2}$. Set

$$
\begin{aligned}
G^{*}\left(t, z, z^{\prime}, \varepsilon\right) & =F\left(t, y^{*}(t), y^{*^{\prime}}(t), \varepsilon\right) \\
& -F\left(t, y^{*}(t)+z, y^{*^{\prime}}(t)+z^{\prime}, \varepsilon\right) \\
& +F_{y}\left(t, y^{*}(t), y^{*^{\prime}}(t), \varepsilon\right) z+F_{y}^{\prime}\left(t, y^{*}(t), y^{*^{\prime}}(t), \varepsilon\right) z^{\prime}
\end{aligned}
$$

for 


$$
|z|<\frac{\delta}{2}, \quad\left|z^{\prime}\right|<\frac{\delta}{2}\left(1+\frac{1}{\varepsilon} e^{-(\phi(t) / \varepsilon)}\right) .
$$

Then

$$
G^{*}(t, 0,0, \varepsilon)=0
$$

and

$$
\begin{aligned}
\mid G^{*}\left(t, z_{1}, z_{1}^{\prime}, \varepsilon\right) & -G^{*}\left(t, z_{2}, z_{2}^{\prime}, \varepsilon\right) \mid \\
& \leqq k \sigma\left(z_{1}, z_{2}, z_{1}^{\prime}, z_{2}^{\prime}\right)
\end{aligned}
$$

as before.

Assume furthermore that

$$
y^{*}(t)=u(t)+O(\varepsilon), \quad y^{*}(t)=u^{\prime}(t)+O(\varepsilon)
$$

and set

$$
\begin{aligned}
& p^{*}(t, \varepsilon)=F_{y^{\prime}}\left(t, y^{*^{\prime}}(t), y^{*^{\prime}}(t), \varepsilon\right)=\phi^{\prime}(t)+\varepsilon p_{1}^{*}(t, \varepsilon) \\
& q^{*}(t, \varepsilon)=F_{\nu}\left(t, y^{*}(t), y^{*^{\prime}}(t), \varepsilon\right) .
\end{aligned}
$$

Then $p^{*}-p$ and $q^{*}-q$ are continuous functions of $t, p^{*}(t, \varepsilon)-p(t, \varepsilon)$ $=\tilde{F}_{y y^{\prime}}\left(y^{*}(t)-u(t)\right)+\tilde{F}_{y^{\prime} y^{\prime}}\left(y^{*^{\prime}}(t)-u^{\prime}(t)\right)=O(\varepsilon)$, and similarly for $q^{*}-q\left(\tilde{F}_{y y}\right.$, etc. are intermediate values of the partial derivatives), and it follows that $p^{*}$ and $q^{*}$ satisfy the conditions imposed on $p$ and $q$ in section 2 , with $\phi^{\prime}$ defined in (4.1).

\section{The construction of $v$}

We now set

$$
y^{*}=u+v
$$

and subject $y^{*}$ to the differential equation (1.1) and the boundary conditions $y^{*^{\prime}}(0)=u^{\prime}(0), y^{*}(1)=\beta(\varepsilon)$. The differential equation for $v$ may be written as

$$
\varepsilon v^{\prime \prime}+p(t, \varepsilon) v^{\prime}+q(t, \varepsilon) v=G\left(t, v, v^{\prime}, \varepsilon\right)
$$

where $p, q, G$ are defined in assumption (C) and (5.1). We also have the boundary conditions

$$
v^{\prime}(0)=0, \quad v(1)=\lambda=\beta(\varepsilon)-\beta(0)
$$

for $v$. With the definition (3.7) of $T_{1}, v$ must then be a solution of the integrodifferential equation $v=T_{1} G$ in which we take $\lambda=\beta(\varepsilon)-\beta(0)$.

The integro-differential equation $v=T_{1} G$ can be solved by successive approximations. Set $v_{-1}(t)=0$, and $v_{n}=T_{1} G_{n-1}$ for $n=0,1,2, \cdots$, where 


$$
G_{n}(t)=G\left(t, v_{n}(t), v_{n}^{\prime}(t), \varepsilon\right) \quad n=-1,0,1,2, \cdots
$$

By (5.3), $G_{-1}(t)=G(t, 0,0, \varepsilon)=O(\varepsilon)$, and it follows from (3.9) that $v_{0}(t)=T_{1} G_{-1}(t)$ and $v_{0}^{\prime}(t)$ are $O(\varepsilon)$. Suppose

$$
\left|v_{0}(t)\right|,\left|v_{0}^{\prime}(t)\right| \leqq A \varepsilon
$$

for some $A$, set $2 A A_{5} k=B$, where $A_{5}$ and $k$ are the constants appearing in (3.9) and (5.4), and assume

$$
0<\varepsilon<\min \left(\frac{\delta}{2 A}, \frac{1}{2 B}, \varepsilon_{0}\right)=\varepsilon_{1} .
$$

We wish to prove by induction that

$$
\begin{aligned}
& \left|v_{n}(t)\right|,\left|v_{n}^{\prime}(t)\right| \leqq 2 A \varepsilon \\
& \left|v_{n}(t)-v_{n-1}(t)\right|, \quad\left|v_{n}^{\prime}(t)-v_{n-1}^{\prime}(t)\right| \leqq A \varepsilon(B \varepsilon)^{n} .
\end{aligned}
$$

Now, clearly (6.6) holds for $n=0$. Suppose that it holds for $n=0,1, \cdots$, $k$ and prove that it holds also for $n=k+1$. Since $2 A \varepsilon<\delta$, $\left(t, u(t)+v_{n}(t), u^{\prime}(t)+v_{n}^{\prime}(t), \varepsilon\right)$ is in $D_{\delta}$ for $n=k-1, k$, and we may form $G_{k-1}, G_{k}$. Moreover, by (5.4) and the induction assumption, $\left|G_{k}(t)-G_{k-1}(t)\right| \leqq 2 A k \varepsilon A \varepsilon(B \varepsilon)^{k}$, and by $(3.9)\left|v_{k+1}(t)-v_{k}(t)\right| \leqq \| T_{1}\left(G_{k}-\right.$ $\left.G_{k-1}\right) \| \leqq 2 A A_{5} k \varepsilon . \quad A \varepsilon(B \varepsilon)^{k}=A \varepsilon(B \varepsilon)^{k+1}$, with the same estimate for $\left|v_{k+1}^{\prime}(t)-v_{k}^{\prime}(t)\right|$, so that the second line of $(6.6)$ holds also for $n=k+1$. Finally,

$$
\left|v_{k+1}(t)\right| \leqq \sum_{n=0}^{k+1}\left|v_{n}(t)-v_{n-1}(t)\right| \leqq \sum_{n=0}^{\infty} A \varepsilon(B \varepsilon)^{n}<2 A \varepsilon
$$

since $B \varepsilon<1 / 2$, and the same estimate holds for $v_{k+1}^{\prime}(t)$.

It follows from (6.6) that

$$
v(t)=\sum_{n=0}^{\infty}\left[v_{n}(t)-v_{n-1}(t)\right]=\lim _{k \rightarrow \infty} v_{k}(t)
$$

defines a continuously differentiable function $v$.

$$
\lim _{k \rightarrow \infty} G_{k}(t)=G\left(t, v(t), v^{\prime}(t), \varepsilon\right)
$$

uniformly for $0 \leqq t \leqq 1$ by continuity of $G$ in $v, v^{\prime}$ and uniform convergence of $v_{k}$ to $v$ and of $v_{k}^{\prime}$ to $v^{\prime}$. Again by uniform convergence, $T_{1} G_{k} \rightarrow T_{1} G$, so that $v$ satisfies the integro-differential equation $v=T_{1} G$ and hence also the differential equation (6.2) and the boundary conditions (6.3).

With this $v, y^{*}=u+v$ is a solution of the differential equation (1.1) that satisfies the second boundary condition (1.2). It follows from (6.6) that the graph of this solution is not only in $D_{\delta}$ but in the narrower region $D_{21 \varepsilon}$. 


\section{The construction of $w$}

Let

$$
0<\varepsilon<\min \left(\frac{\delta}{4 A}, \frac{1}{2 B}, \varepsilon_{0}\right)=\varepsilon_{2},
$$

and let $y^{*}$ be that solution of (1.1) constructed in section 6. By the remark made at the end of section 6 , the graph of this solution is in $D_{\delta / 2}$.

Writing the solution of $P_{\varepsilon}$ in the form

$$
y=y^{*}+w,
$$

and using the notations (5.5) and (5.10), we may write the differential equation for $w$ in the form

$$
\varepsilon w^{\prime \prime}+p^{*}(t, \varepsilon) w^{\prime}+q^{*}(t, \varepsilon) w=G^{*}\left(t, w, w^{\prime}, \varepsilon\right),
$$

while the boundary conditions for w read

$$
w(0)=\alpha(\varepsilon)-u(0)-v(0)=\mu, \text { say; } w(1)=0 .
$$

Since $y^{*}$ satisfies (5.9), $p^{*}$ and $q^{*}$ satisfy the conditions imposed on $p$ and $q$ in section 4. Also $p^{*}(t, 0)=\phi^{\prime}(t)$. We shall denote by $V_{3}^{*}$ the function corresponding to $V_{3}$ when $p$ and $q$ are replaced by $p^{*}$ and $q^{*}$, and by $T_{3}^{*}$ the operator corresponding to $T_{3}$. From (3.4) we conclude the existence of a constant $A^{*}$ such that

$$
\left|e^{\phi(t) / \varepsilon} V_{3}^{*}(t)\right|, \varepsilon\left|e^{\phi(t) / \varepsilon} V_{3}^{* \prime}(t)\right| \leqq A^{*} .
$$

The constant corresponding to $A_{6}$ in $(3.10)$ will be denoted by $A_{6}^{*}$, and $2 A^{*} A_{\mathbf{6}}^{*} k=B^{*}$.

Any solution of the integro-differential equation $w=T_{3}^{*} G^{*}$ satisfies (7.3) and (7.4), and it will be shown that under a certain restriction on $\mu$, such a solution can be constructed by successive approximations, setting $w_{-1}(t)=0$ and, in a notation similar to (6.4),

$$
w_{n}=T_{3}^{*} G_{n-1}^{*} \text { for } n=0,1,2, \cdots .
$$

It follows from (5.7), (3.7) and (7.5) that

$$
\left|e^{\phi(t) / \varepsilon} w_{0}(t)\right|, \varepsilon\left|e^{\phi(t) / \varepsilon} w_{0}^{\prime}(t)\right| \leqq A^{*}|\mu| .
$$

If we now assume that

$$
|\mu|<\min \left(\frac{\delta}{4 A^{*}}, \frac{1}{2 B^{*}}\right)=\mu_{1}
$$

then the appraisals 


$$
\begin{aligned}
& e^{\phi(t) / \varepsilon}\left|w_{n}(t)\right|, \varepsilon e^{\phi(t) / \varepsilon}\left|w_{n}^{\prime}(t)\right| \leqq 2 A^{*}|\mu| \\
& e^{\phi(t) / \varepsilon}\left|w_{n}(t)-w_{n-1}(t)\right|, \varepsilon e^{\phi(t) / \varepsilon}\left|w_{n}^{\prime}(t)-w_{n-1}^{\prime}(t)\right| \leqq A^{*}|\mu|\left(B^{*}|\mu|\right)^{n}
\end{aligned}
$$

can be proved by induction. The proof parallels that of (6.6), except that (5.8) and (3.10) are used in place of (5.4) and (3.9).

$$
w(t)=\lim _{k \rightarrow \infty} w_{k}(t)=\sum_{n=0}^{\infty}\left[w_{n}(t)-w_{n-1}(t)\right]
$$

defines a differentiable function $w$ that satisfies the integro-differential equation $w=T_{3}^{*} G^{*}$, and hence also the differential equation (7.3) and the boundary conditions (7.4).

By (7.6) and (7.7),

$$
|w(t)|, \varepsilon\left|w^{\prime}(t)\right| \leqq 2 A^{*}|\mu| e^{-(\phi(t)) \varepsilon)}<\frac{\delta}{2} e^{-(\phi(t) / \varepsilon)}
$$

so that the graph of $y=y^{*}+w=u+v+w$ lies in $D_{\jmath}$, and $y$ satisfies the differential equation (1.1) and the boundary conditions (1.2).

Now let $\mu_{1}=2 \mu_{0}$, and assume that

$$
0<\varepsilon<\min \left(\varepsilon_{2}, \frac{\mu_{0}}{2 A}\right)=\varepsilon_{3}, \quad|\alpha(\varepsilon)-u(0)|<\mu_{0} .
$$

Since $|v(t)| \leqq 2 A \varepsilon<\mu_{0}$ in this case, we have $|\mu|=|\alpha(\varepsilon)-u(0)-v(0)|$ $<2 \mu_{0}=\mu_{1}$ so that the result established above is valid. $y$ is a solution of $P_{\varepsilon}$, and the assertions of the theorem of section 4 are proved as far as the existence of the solution and the appraisals of $v$ and $w$ are concerned.

\section{Uniqueness of the solution}

We now make the further assumption

$$
\left|F_{y}\left(t, y, y^{\prime}, \varepsilon\right)\right| \leqq A, \quad F_{v^{\prime}}\left(t, y, y^{\prime}, \varepsilon\right) \geqq B>0
$$

in $D_{\delta}$ and wish to prove that in this case $P_{\varepsilon}$ possesses at most one solution in $D_{\delta}$.

Let us denote by $y=y(t, \varepsilon, \gamma)$ that solution of the differential equation (1.1) satisfying the initial conditions

$$
y(0)=\alpha(\varepsilon), \quad y^{\prime}(0)=\gamma .
$$

Here $\gamma$ may depend on $\varepsilon$, and we must have

$$
\left|\gamma-u^{\prime}(0)\right|<\delta\left(1+\frac{1}{\varepsilon}\right)
$$

to ensure that $\left(t, y(t), y^{\prime}(t), \varepsilon\right)$ is in $D_{\delta}$ at least for sufficiently small $t$. It 
follows from the basic theorems on the continuation of solutions of differential equations and on their dependence on initial conditions, that as long as the solution $y(t, \varepsilon, \gamma)$ remains in $D_{\delta}$, it is unique and is a differentiable function of $\gamma$. Moreover,

$$
z=\frac{\partial y(t, \varepsilon, \gamma)}{\partial \gamma}
$$

satisfies the "variational equation"

$$
\varepsilon z^{\prime \prime}+f(t) z^{\prime}+g(t) z=0
$$

in which

$$
f(t)=F_{y^{\prime}}\left(t, y(t), y^{\prime}(t), \varepsilon\right), \quad g(t)=F_{y}\left(t, y(t), y^{\prime}(t), \iota\right) .
$$

In addition, $z$ satisfies the initial conditions

$$
z(0)=0, \quad z^{\prime}(0)=1 .
$$

We shall prove that under our conditions,

$$
z(t)>0 \text { for } 0<t \leqq 1
$$

provided that $\varepsilon$ is sufficiently small.

To prove (8.5), we use Prüfer's transformation, introducing polar coordinates $(r, \theta)$ in the phase plane; and setting

Differentiating

$$
z=r \sin \theta, \quad \varepsilon z^{\prime}=r \cos \theta .
$$

$$
\varepsilon z^{\prime} \sin \theta-z \cos \theta \neq 0
$$

with respect to $t$, and using (8.3), we obtain

$$
\varepsilon \theta^{\prime}=(f \sin \theta+\cos \theta) \cos \theta+\varepsilon g \sin ^{2} \theta .
$$

For sufficiently small $\varepsilon$ there exist $\theta_{1}$ and $\theta_{2}$ such that $0<\theta_{1}<\pi / 2<\theta_{2}$ $<\pi$ and, from (8.6), $\theta^{\prime}>0$ when $0 \leqq \theta \leqq \theta_{1}$, while $\theta^{\prime}<0$ when $\theta=\theta_{2}$. The solution $z$ of (8.3) starts at $r=\varepsilon, \theta=0$. As long as the graph of the solution in the phase plane is in the sector $0 \leqq \theta \leqq \theta_{1}$, we have $\theta^{\prime}>0$, and once the solution enters the sector $\theta_{1} \leqq \theta \leqq \theta_{2}$, it cannot leave it. Thus, $0<\theta<\pi$ for $0<t \leqq 1$, and (8.5) follows.

We have now proved that

$$
z(1)=\frac{\partial y(1, \varepsilon, \gamma)}{\partial \gamma}>0
$$

for sufficiently small $\varepsilon$, so that $y(1, \varepsilon, \gamma)$ is a strictly increasing function of $\gamma$ in this case. It follows that for sufficiently small $\varepsilon$ there is at most one value of $\gamma=\gamma(\varepsilon)$ such that $\gamma(1, \varepsilon, \gamma(\varepsilon))=\beta(\varepsilon)$, and this completes the proof of uniqueness. 


\section{References}

[1] Coddington, E. A. and Levinson, N., A boundary value problem for a nonlinear differential equation with a small parameter. Proc. Amer. Math. Soc. 3 (1952), 73-81.

[2] Wasow, W., Singular perturbations of boundary value problems for nonlinear differential equations of the second order. Comm. Pure Appl. Math. 9 (1956), 93-113.

[3] Briš, N. I., On boundary problems for the equation $\varepsilon y^{\prime \prime}=f\left(x, y, y^{\prime}, \varepsilon\right)$ for small $\varepsilon$ 's. (Russian) Dokl. Akad. Nauk SSSR (N. S.) 95 (1954) $429-432$.

California Institute of Technology

Pasadena, California, U.S.A. 